\title{
SyncGen: An Aspect-Oriented Framework for Synchronization
}

\author{
Xianghua Deng, Matthew Dwyer, John Hatcliff, and Masaaki Mizuno \\ Department of Computing and Information Sciences \\ Manhattan, KS 66506, USA \\ \{deng, dwyer, hatcliff, masaaki\}@cis.ksu.edu
}

\begin{abstract}
This paper describes SyncGen - a tool for automatically synthesizing complex synchronization implementations from formal high-level specifications. In SyncGen, synchronization specifications are phrased using first-order logic or user-friendly specification patterns. From a high-level specification, a language independent synchronization solution in an intermediate guarded-command language is synthesized. Back-end translators can translate this intermediate solution into a variety of implementation frameworks including Java, C++/C with POSIX threads, and Controller Area Network message passing primitives. SyncGen has been used extensively in courses at Kansas State University. Its breadth of applicability has been demonstrated by using it to solve virtually all of the exercises given in the well-known concurrency text books of Andrews [1|2] and Hartley [4], as well as a variety of real-world problems in the embedded computing domain. The tool, along with supporting documentation and an example repository, is publicly available [6].
\end{abstract}

Concurrency is a fundamental tool for meeting the ever increasing performance demands placed on software. With improved performance, however, comes the risk of unintended interference between software components executing in parallel. Developing robust synchronization policies and implementations that assure correct component collaboration without unduly restricting parallelism is a significant challenge. Inspired by Andrews' global invariant approach to synchronization generation [1], we have developed SyncGen to allow application developers to synthesize customized synchronization solutions without having to concern themselves with the low-level details of their implementation. SyncGen separates the synchronzation and functional implementation of an application and provides aspect-oriented support for weaving them into a complete system. Using SyncGen, users develop sequential application code and identify regions of the application that are inter-dependent. The boundaries of those regions form the (cut) points at which synchronization policies must be enforced. Users can give a high-level formal specification of the synchronization policy which governs the collaborative execution of a group of code regions and SyncGen automatically synthesizes efficient synchronization aspect code and weaves that synchronization code into the core functional code at the appropriate region boundaries. The toolset is:

- Useful - the tool has been used in advanced operating systems courses extensively at Kansas State University, and students and instructors find it tremendously useful. 


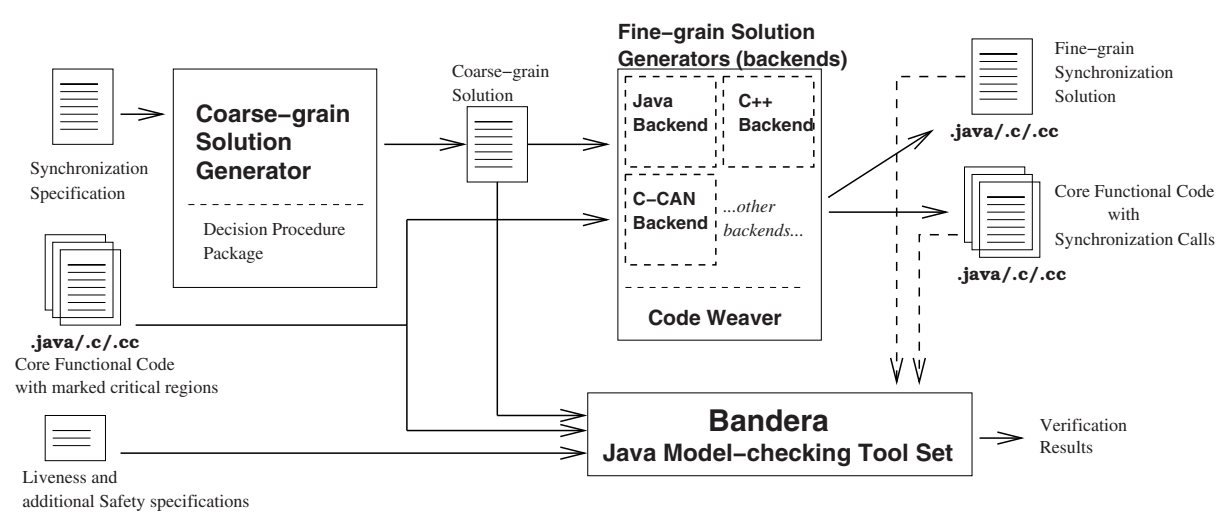

Fig. 1. SyncGen tool architecture

- Powerful - using a synchronization pattern system, complicated policies can be described clearly and succinctly at a very high level.

- Expressive - we have used the pattern system to specify solutions for almost all exercises from two well-known concurrency texts [1 2,4].

- Automatic - this is a push-button approach where code with very intricate semantics is automatically generated from high-level specifications. Thus, it is very easy to learn to use the tool.

- General - the approach is language independent and supports multiple target languages and synchronization primitives. (e.g., we currently have full tool support implemented for Java, C/C++ with POSIX threads).

- Formal - our aspect specification language has a rigorous semantic foundation which enables code generation techniques that use decision procedures for a quantifier-free fragment of first-order arithmetic and yields a high degree of confidence in the generated solutions.

- Verifiable - the structure of the generated code and associated artifacts is designed so that crucial correctness requirements can be checked automatically using existing software model-checking technology.

The tool architecture in Figure 1 depicts the main components of SyncGen, the artifacts that the user supplies (on the left), and the artifacts that the toolset synthesizes (on the right). These artifacts are discussed as we explain the operation of the tool below; for a more complete presentation see [3].

Using the SyncGen approach to developing concurrent software, a developer uses traditional methods and development environments to produce what we term the system's core functional code. This code realizes the behavior of each concurrent component of the system, but does not specify how components synchronize. Instead, the developer simply marks the regions of code in each component that require synchronization with syntactic tags. The developer then partitions regions into equivalence classes termed clusters based on which regions refer to each other. Intuitively, regions $R_{1}$ and $R_{2}$ should be in the same cluster if a thread at region $R_{1}$ waits for an event or state that is triggered or changed by a thread at $R_{2}$.

For example, consider a web server program in which a query request writes information into a shared buffer $b$ and an update request reads the information in $b$. At 
run-time, the server instantiates a new thread for each query and update request. To guarantee consistency of the buffer information, the program imposes the well-known readers/writers synchronization policy, in which only one thread may update the buffer but concurrent threads may read the buffer. Then, within the functional code for the query request, there is a synchronization region $R_{r}$ in which threads read from $b$. Similarly, the functional code for the update request has a synchronization region $R_{w}$ in which threads write into $b$. Regions $R_{r}$ and $R_{w}$ form a sole cluster $C_{r w}$ in the program.

Once synchronization regions and clusters are identified, the developer constructs a SyncGen synchronization specification that formally states the safety property of each cluster in terms of an invariant defined over region occupancy. Let $n r$ and $n w$ be the number of threads currently reading and writing $b$, respectively. Then, an appropriate invariant $I$ for $C_{r w}$ is $(n r=0 \vee n w=0) \wedge(n w \leq 1)$.

Given this high-level global invariant, SyncGen automatically generates a coarsegrained synchronization solution for each cluster. Notice that we must guarantee that $n r$ and $n w$ correctly keep track of the number of reading threads and writing threads, respectively. Therefore, before a query (update) thread enters region $R_{r}\left(W_{r}\right), n r(n w)$ must be incremented by one. Similarly, after a query (update) thread exits $R_{r}\left(R_{w}\right), n r$ ( $n w$ ) must be decremented by one. A coarse-grained solution performs the increments so as to maintain $I$, using the following two high-level synchronization constructs:

1. $\langle S\rangle$ : Statement $S$ is executed atomically.

2. $\langle$ await $B \rightarrow S\rangle$ : Atomically check if $B$ is true, then execute $S$. If $B$ is false, the executing thread waits until $B$ becomes true and executes $S$.

For the execution of $n r++$, the following Hoare triple must hold: $\{I\}\langle$ await $B \rightarrow$ $n r++\rangle\{I\}$; this says that if $I$ holds in a state where $B$ holds, then executing $n r++$ will result in a state in which $I$ holds. Logically $B$ can be mechanically derived as the weakest condition such that $I \wedge B \Rightarrow w p(n r++, I)$, where $w p(n r++, I)$ denotes the weakest condition that may hold and still guarantee that if $n r++$ executes $I$ will hold. If $B$ is true, $\langle S\rangle$ is used. For the web site program, the following coarse-grained solution is derived.

$$
\begin{aligned}
& \langle\text { await } n w=0 \rightarrow n r++\rangle \\
& \quad \text { read from B } \\
& \langle n r--\rangle
\end{aligned}
$$$$
\langle\text { await } n w=0 \wedge n r==0 \rightarrow n w++\rangle
$$$$
\text { write into } \mathrm{B}
$$$$
\langle n w--\rangle
$$

Translation algorithms exist from a coarse-grained solution to various languages and synchronization primitives (called fine-grained code), including translations to Java, POSIX P-thread, CAN, active monitor, and semaphores. Each translation has been proved to maintain the global invariant; therefore, the resulting code satisfies the synchronization specification.

The functional code and fine-grained synchronization aspect code are woven by inserting, at the entries to and exits from synchronization regions in the functional code, call statements to the corresponding aspect code.

SyncGen automates the above processes (1) to generate a coarse-grained solution from a global invariant, (2) to translate the coarse-grained solution to fine-grained synchronization code, and (3) to weave the function code and the aspect code. Currently, 
SyncGen implements translation algorithms to Java synchronization blocks and POSIX P-threads.

SyncGen logical specifications of invariants that capture synchronization policies. To make SyncGen more broadly usable by software developers, we have identified a large number of commonly occurring synchronization patterns and presented them to users as parameterized templates that are invoked by name. Some of the most commonly used patterns are:

- $\operatorname{Bound}(R, n)$ : A cluster consists of a single synchronization region $R$. At most $n$ threads can be in $R$.

- Exclusion $\left(R_{1}, R_{2}, \cdots, R_{n}\right)$ : A cluster consists of $n$ regions $R_{1}, R_{2}, \cdots, R_{n}$. Threads can be in at most one synchronization region out of the $n$ synchronization regions.

- $k$-MuTex $\left(R_{1}, R_{2}, \cdots, R_{n}, k\right)$ (k-Mutual Exclusion): At most $k$ threads can be in regions in the cluster.

- Barrier $\left(\left(R_{1}, N_{1}\right),\left(R_{2}, N_{2}\right), \cdots,\left(R_{n}, N_{n}\right)\right): N_{i}$ number of threads entering region $R_{i}$ for $1 \leq i \leq n$ meet, form a group, and leave the respective synchronization regions together.

As variations of the Barrier pattern, we have

- Asymmetric Barrier: an asymmetric version of the Barrier pattern, where threads entering a subset of regions trigger the departure of threads from another subset of regions.

- Barrier with Information Exchange: An extension of the Barrier pattern where the threads forming a group exchange information before leaving together.

For example, the previous readers/writers synchronization may be specified by $\operatorname{Bound}(W, 1) \wedge \operatorname{Exclusion}(R, W)$. We have successfully solved most synchronization problems found in several advanced operating systems textbooks. For example, the Sleeping Barber problem may be solved by using the Barrier (for the barber and a customer to meet), $k$-Mutex (for customers to sit/leave chairs in the waiting room), and Asymmetric Barrier (for the barber to notify the customer of completion of the hair cut) patterns. The multiple barbers problem may be solved by simply changing the Barrier pattern to the Barrier with Information Exchange pattern so that a customer can remember which barber cuts his hair. We have also successfully applied SyncGen and the patterns to implement various event-triggered embedded control systems. In such systems, we found that the Asymmetric Barrier and Bound patterns were most used in sending/receiving events and forming critical sections, respectively.

The SyncGen web site [6] contains much more information on the usage of our tool. The complete catalog of examples, a repository of examples, a tutorial on how to use SyncGen and the implementation which synthesizes Java implementations are all available for public download. 


\section{References}

1. G. R. Andrews. Concurrent Programming: Principles and Practice. Addison-Wesley, 1991.

2. G. R. Andrews. Foundations of Multithreaded, Parallel, and Distributed Programming. Addison-Wesley, 2000.

3. X. Deng, M. Dwyer, J. Hatcliff, and M. Mizuno. Invariant-based specification, synthesis, and verification of synchronization in concurrent programs. In ICSE, 2002.

4. S. Hartley. Concurrent Programming - The Java Programming Language. Oxford University Press, 1998.

5. M. Mizuno. A structured approach for developing concurrent programs in Java. Information Processing Letters, 69(5):233-238, Mar. 1999.

6. X.Deng, M. Dwyer, J. Hatcliff, and M. Mizuno.

SyncGen. |1]ttp://syncgen.projects.cis.ksu.edu|, 2002. 